\title{
Fibromatosis colli in a neonate- A case report
}

\author{
Rajkumar S Yalawar ${ }^{1}$, Shashikiran B R ${ }^{2}$, Ganesha Shetty ${ }^{2}$, B S Prasad ${ }^{3}$ \\ ${ }^{I}$ Associate Professor, Department of Radiology, SSIMS \& RC, Davangere, Karnataka, India. \\ ${ }^{2}$ Postgraduate student, Department of Radiology, SSIMS \& RC, Davangere, Karnataka, India. \\ ${ }^{3}$ Professor, Department of Pediatrics, SSIMS \& RC, Davangere, Karnataka, India.
}

\begin{abstract}
Fibromatosis colli or pseudotumor of infancy of the sternocleidomastoid muscle is a rare cause of a benign neck mass in neonates and infants. Though exact etiology is not known, it is most likely due to birth trauma. Ultrasonography is the imaging modality of choice and nearly all the cases are correctly diagnosed if clinical and radiologically suspected. This entity is a self-limiting condition and resolves over a period of time. If diagnosed correctly can be managed conservatively and prevent unnecessary diagnostic and therapeutic maneuvers. We report a rare case of fibromatosis colli in a 1 month old neonate diagnosed using ultrasonography.
\end{abstract}

Keywords: Fibromatosis colli, neonate, pseudotumor, sternocleidomastoid muscle

\section{Introduction}

Fibromatosis colli or pseudotumor of infancy of the sternocleidomastoid muscle is a rare benign cause of neck swelling or mass in neonate and infants. Though the exact etiology is not known, it is most likely due to birth trauma or malposition in uterus. It is one of the cause of congenital torticollis and usually recognized by mother as neck swelling with or without restricted neck movement. Ultrasonography of neck is the imaging modality of choice and sometimes CT scan or MRI scan may be required to further characterize the disease and extent of involvement. Real time ultrasonography demonstrate synchronous motion of the mass with the sternocleidomastoid muscle, thus confirming the diagnosis.

We present a case report of fibromatosis colli in a 1 month old neonate diagnosed using ultrasonography. In addition to this brief discussion was made on literature review, clinical and radiological findings and differentials.

\section{Case Report}

A 4 week old neonate was referred to the radiology department for ultrasonography of a neck swelling on the right side which was incidentally noticed by the mother 1 week ago. On examination the neck swelling was firm, partially mobile, no warm on touch and approximately $1.5-2 \mathrm{~cm}$ soft tissue mass was felt on the right side of neck (figure 1). Neonate was afebrile. There was restriction of neck movements on the affected side and chin is directed away. Past history revealed difficult labour and birth asphyxia, however no assisted delivery was noted. Baby did not cry immediately after birth and was admitted for three days in neonatal intensive care unit. Later baby started feeding well and was immunized till date. Ultrasonography of neck showed ill-defined fusiform thickening of right distal sternocleidomastoid muscle with heterogeneous echotexture (figure 2). The fibrillar structure of muscle fibers are well maintained. On color doppler study, minimal peripheral vascularity with high resistance flow is noted (figure 3). No significant cervical nodes or vascular engorgement was noted. Left sternocleidomastoid muscle was normal in bulk and signal intensity. In view of clinical history and ultrasonography findings a diagnosis of fibromatosis colli or pseudotumor of the sternocleidomastoid muscle was considered. The condition was managed conservatively. Physiotherapy and neck stretching exercises were started. The neck swelling showed slight decrease in sized after 4 week with near normal neck movements. 


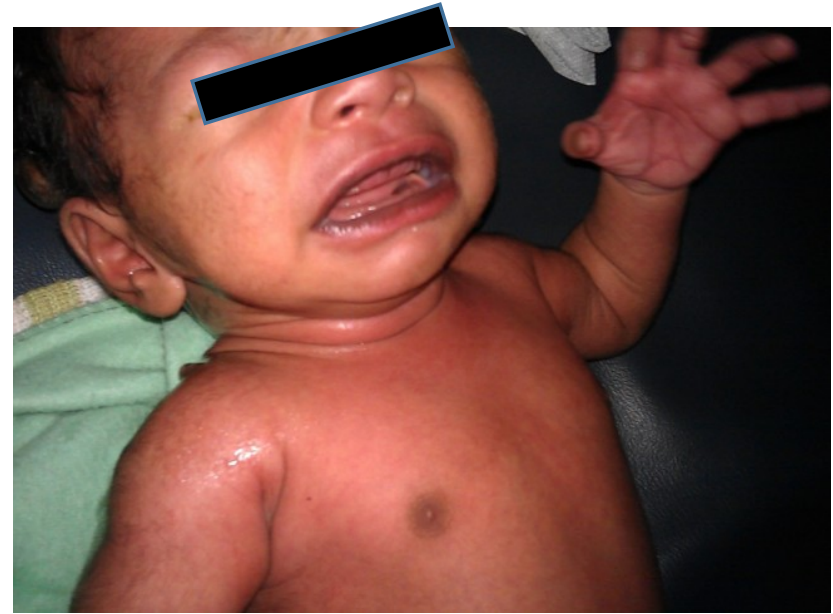

Figure 1: 4 week old neonate showing a neck swelling on right side and slight restriction of neck movement
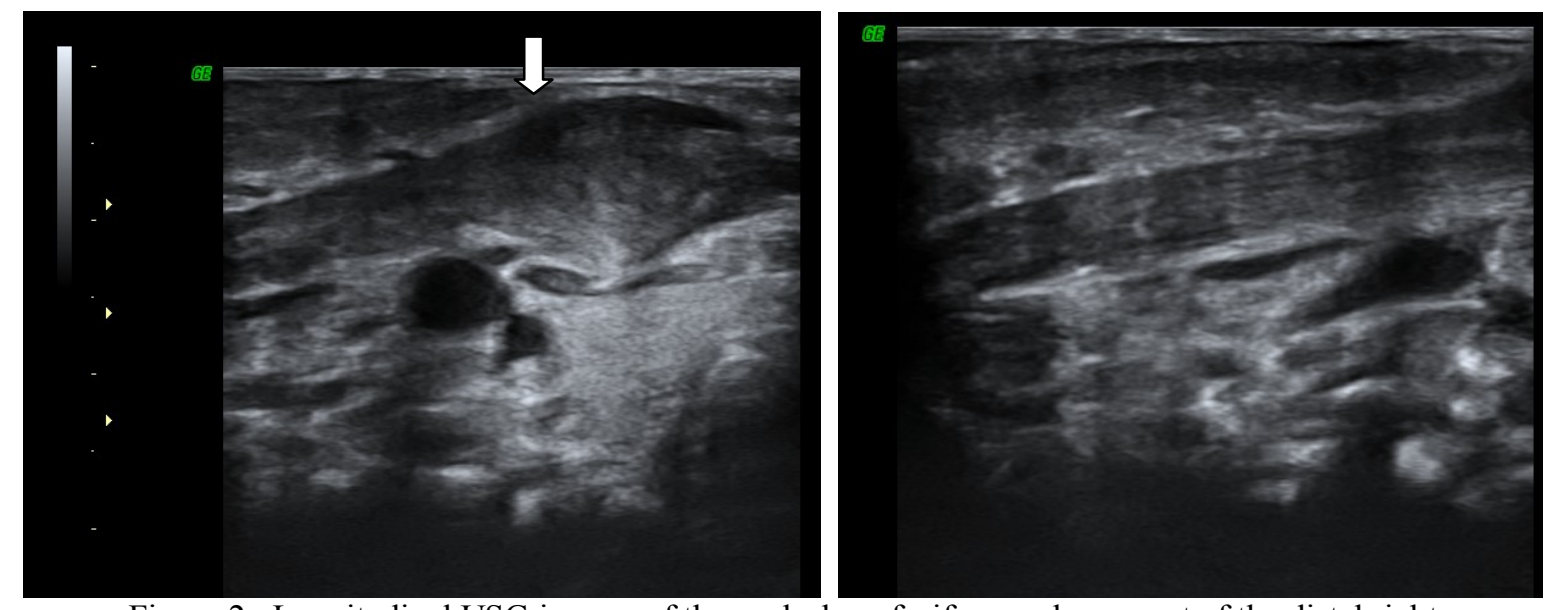

Figure 2: Longitudinal USG images of the neck show fusiform enlargement of the distal right sternocleidomastoid muscle (right) and normal muscle on left side (left).
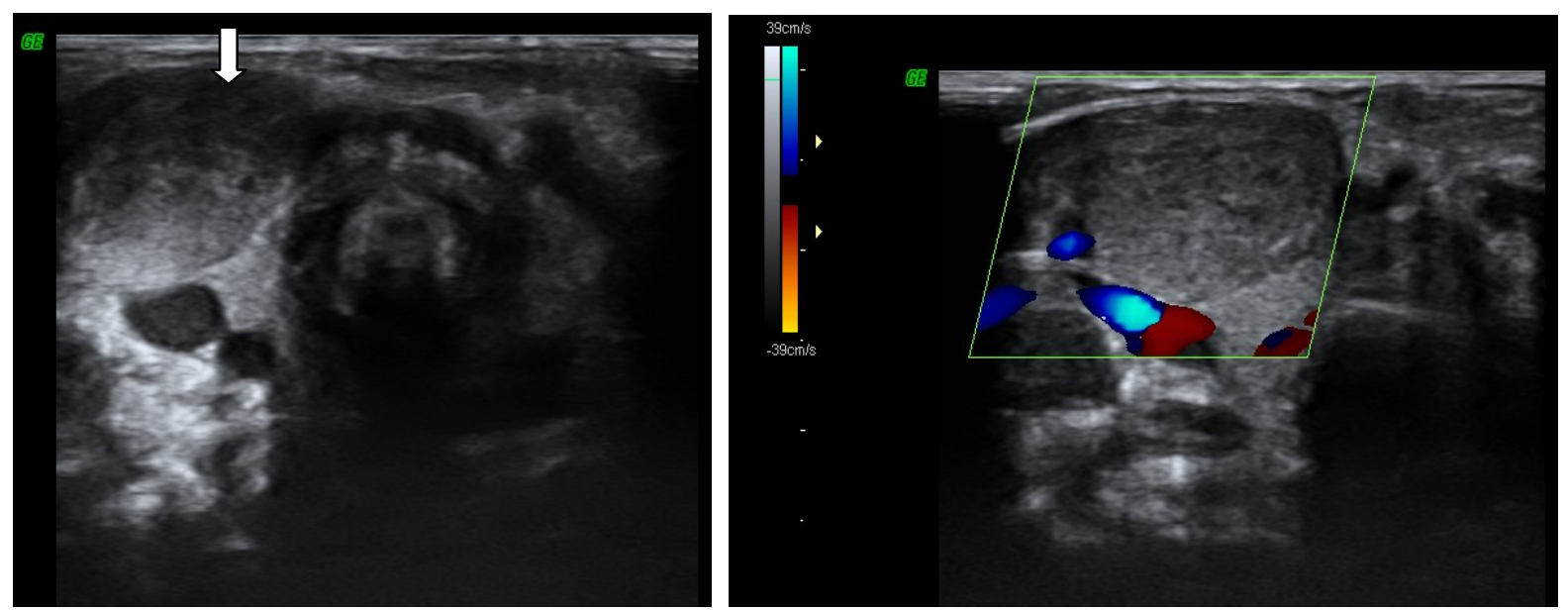

Figure 3: Transverse USG images of the neck shows oval soft tissue mass at distal right sternocleidomastoid muscle with minimal peripheral vascularity (right) and normal bulk in the left sternocleidomastoid muscle (left). 


\section{Discussion}

Fibromatosis colli is a rare benign condition in neonates and infants with neck swelling. Most cases show no abnormality at birth but manifest between the 2nd and 4th weeks of life as a firm soft-tissue mass in the lower one-third of the sternocleidomastoid muscle ${ }^{1,6}$. It is usually unilateral, slightly more common on the right side while bilateral involvement is rare. It affects both the sternal and clavicular heads of the muscle and seen more commonly in male child ${ }^{1}$. About $14-20 \%$ cases of congenital torticollis are due to contraction of the sternocleidomastoid muscle ${ }^{2}$. The exact cause is unclear, it is likely related to birth trauma greater than $90 \%$ of cases associated with a difficult or forceps delivery. Some cases are due to abnormal utero fetal head position, which causes selective injury and leads to development of a secondary compartment syndrome, pressure necrosis and fibrosis within the sternocleidomastoid muscle ${ }^{2}$. No cervical lymphadenopathy, vascular invasion or bony involvement are seen as compared other neck masses.

Ultrasonography (USG) is the best imaging modality for diagnosis due to its relative low cost, its lack of radiation and the proximity of the lesion to the skin ${ }^{4}$. USG findings show fusiform thickening or diffusely enlarged sternocleidomastoid muscle with variable echogenicity. Color doppler interrogation may reveal a high resistance waveform. The enlarged area often moves synchronously with the rest of the sternocleidomastoid muscle (SCM) on real time sonography ${ }^{4}$. Almost all the cases can be identified by ultrasonography as compared to CT or MRI. Computed tomography (CT) show a homogeneous diffusely enlarged sternocleidomastoid muscle with surrounding well preserved fat planes. At times calcification may be present ${ }^{5}$. Magnetic resonance imaging (MRI) shows decreased signal intensity of the mass on T2W images as compared to gradient-recalled $\mathrm{T} 1 \mathrm{~W}$ images, because of the presence of fibrous tissue ${ }^{7}$. The extent of involved muscle is better delineated with MRI than with USG. Radiographs are usually normal and sometimes used to exclude congenital bony abnormality. Histological features include bland-appearing fibroblasts, myofibroblasts and atrophic skeletal muscle along with muscle giant cells and bare nuclei ${ }^{8}$.

Fibromatosis colli is self-limiting condition and usually resolves within 4-8 months and requires physiotherapy and neck stretching exercises ${ }^{9}$. Approximately two-third cases regress by the age of 2 years ${ }^{3}$. The differential diagnosis of solid masses in the neck include neuroblastoma, rhabdomyosarcoma, lymphoma and other sarcomas. In addition to neck mass, may have enlarged cervical lymph nodes, vascular encasement, or invasion of surrounding structures. Cystic mass in the neck near the midline includes cystic hygroma, branchial cleft cyst, dermoid cyst, teratoma or thyroglossal duct cyst are considered in the differentials.

\section{Conclusion}

Our case highlights, fibromatosis colli or pseudotumor of infancy of the sternocleidomastoid muscle is a rare benign cause of neck swelling or mass in neonate and infants. The radiologist must be aware of its imaging features in order to differentiate it from other neck masses. This entity is a self-limiting condition and resolves over a period of time. If diagnosed correctly can be managed conservatively and prevent unnecessary diagnostic and therapeutic maneuvers.

\section{Acknowledgements}

My sincere thanks to Dr. Pavitra who helped us in preparing the manuscript.

Conflict of Interest: Nil

Fund support: Nil

\section{References}

[1] Enzinger FM, Weiss SW. Soft tissue tumors 3rd ed. St Louis, Mo: Mosby, 1995

[2] Davids JR, Wenger DR, Mubarak SJ. Congenital muscular torticollis: sequelae of intrauterine or perinatal compartment syndrome. J Pediatr Orthop 1993; 13:141-147.

[3] Coventry MB, Harris LE, Bianco AJ, Bulbulian AH. Congenital muscular torticollis (wryneck). Postgrad Med 1960; $28: 383-391$.

[4] Patrick LE, O'shea P, Simoneaux SF et-al. Fibromatoses of childhood: the spectrum of radiographic findings. AJR Am J Roentgenol. 1996;166 (1): 163-9.

[5] Meuwly JY, Lepori D, Theumann N et-al. Multimodality imaging evaluation of the pediatric neck: techniques and spectrum of findings. Radiographics. 25 (4): 931-48.

[6] Robbin MR, Murphey MD, Temple HT et-al. Imaging of musculoskeletal fibromatosis. Radiographics. 21 (3): 585-600.

[7] Ablin DS, Jain K, Howell L, Steel D. West-Ultrasound and MR imaging of Fibromatosis Colli Pediatr Radiol. 1998; 28:230-3.

[8] Sharma S, Mishra K, Khanna G. Fibromatosis colli in infants- a cytologic study of eight cases. Acta Cytol. 2003; 47:359-62.

[9] Crawford SC, Harnsberger HR, Johnson L et-al. Fibromatosis colli of infancy: CT and sonographic findings. AJR Am J Roentgenol. 1988;151 (6): 1183-4. 